\title{
Optical Free-Space Communication on Earth and in Space regarding Quantum Cryptography Aspects
}

\author{
Christian Fuchs*, Dr. Dirk Giggenbach \\ German Aerospace Center (DLR) \\ \{christian.fuchs, dirk.giggenbach\}@dlr.de
}

\begin{abstract}
Mobile FSO Communication links have gained significant attention over the last years due to their increasing maturity. They are used in point to point link scenarios where the high gain in power efficiency and datarate is crucial, compared to state-of-the-art RF links.

The Optical Communication Group of German Aerospace Center's Institute of Communication and Navigation is conducting research and practical investigations of mobile optical high-speed communication links between diverse platforms, e.g. optical links from aircrafts, UAVs or Satellites to Ground.

This paper will give an overview on the current and future work of the Optical Communication Group in this field, with additional regard to quantum communications.
\end{abstract}

Key words: FSO, Free-Space Optical Communications, Quantum Cryptography

\section{Introduction}

Free-space optical communications for mobile applications have become more and more popular during the last couple of years. Especially in applications that require high datarates and power efficencies, FSO links are a good alternative to state-of-the-art RF links. An additional advantage is the fact that the optical spectrum is unregulated and thus no time-consuming licensing process is necessary.

The Optical Communication Group of German Aerospace Center's Institute of Communication and Navigation is conducting research for a broad variety of possible applications. This includes the demonstration of free-space optical links from stratospheric platforms to ground, aircraft to ground and satellites to ground. Furthermore, challenging topics as e.g. adaptive optics systems for highly turbulent atmospheric channels or deep space links for exploration probes are investigated.

As the design of classical FSO terminals is very similar to quantum communication terminals, it seems feasible to combine both into a single terminal. This approach would enable a number of additional applications, as e.g. the establishment of virtually tap proof communication links. This goal can be achieved 
by using the quantum communication subsystem for exchanging a secure key at the beginning of communication, while transmitting the actual data with a high rate over the classical communication channel.

\section{High Altitude Platform-to-Ground Links}

A very interesting and promising application for free-space optical links is the interconnection of airships placed in the stratosphere, so called High Altitude Platforms (HAPs). Equipped with RF-systems for the connection to users on ground and optical terminals for building up a network among them, HAPs offer a cost-effective solution for offering broadband data access in regions where only little terrestrial infrastructure exists or where it would be too expensive to build it up.

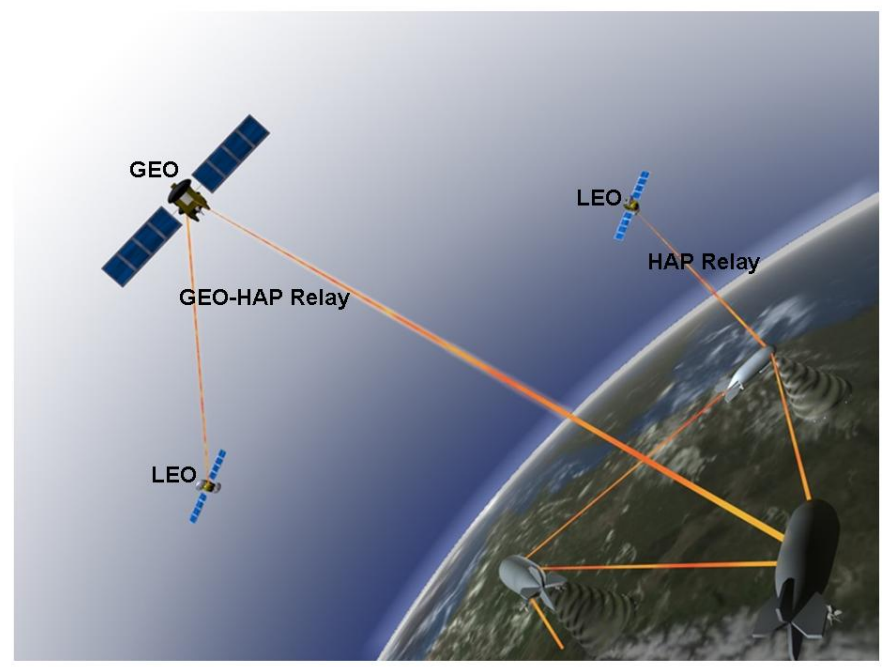

Fig. 1. Artist's Impression of a network including High Altitude Platforms (HAPs) in the stratosphere. Users can connect through weather-insensible RF communication links, while optical Inter-HAP links serve as backbone. Further applications are the use of HAPs as data relay for LEO- and GEO-satellites.

As the operating altitude of HAPs is above the height of clouds, no link blockings for the Inter-HAP-links can be expected due to clouds. At the same time, the RF user links can operate through the clouds, also during bad weather conditions.

Furthermore, HAPs can be used as data-relay for optical downlinks from satellites. As an example, a LEO satellite could dump it's data via a highrate optical link to the HAP during the relatively short contact time of several 
minutes. Subsequently, the data could be forwarded to ground by means of a standard RF link that is not influenced by the weather underneath the HAP [1].

However, the development of optical terminals for HAPs is a challenging task. Virtually no air-pressure and very low temperatures at the operation altitude demand a sophisticated system design, especially if a high durability is required for the equipment.

DLR has developed an FSO terminal for stratospheric applications, and demonstrated its functionality in the framework of the EU funded project CAPANINA $[2,3]$. A downlink from a stratospheric ballon with a height of up to $25 \mathrm{~km}$ with a data rate of $1,25 \mathrm{Gbit} / \mathrm{s}$ over a distance of $64 \mathrm{~km}$ has been performed. Figure 2 shows pictures of the development. A periscope-type Coarse Pointing Assembly (CPA) was used for the terminal.
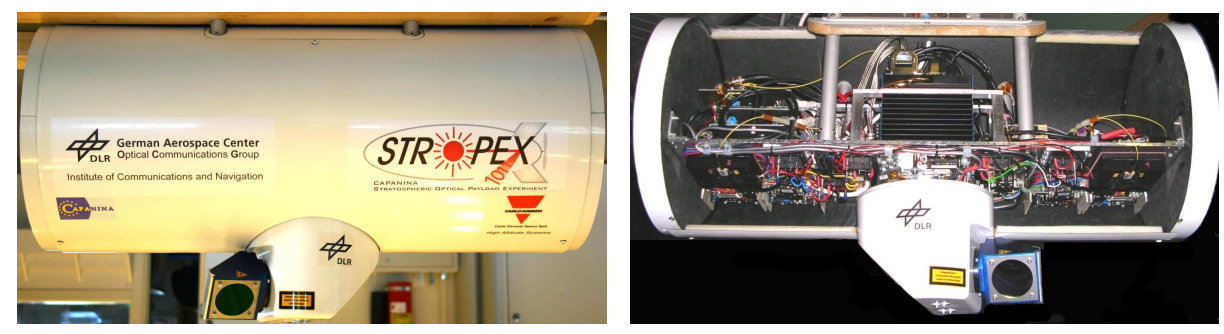

Fig. 2. Freespace Experimental Laser Terminal (FELT) that has been developed for the CAPANINA Trial

The validation trial was carried out at the ESRANGE facilities in Kiruna, Sweden. Figure 3 shows a picture of the ballon launch. The payload was mounted in a compartment at the bottom of the ballon.

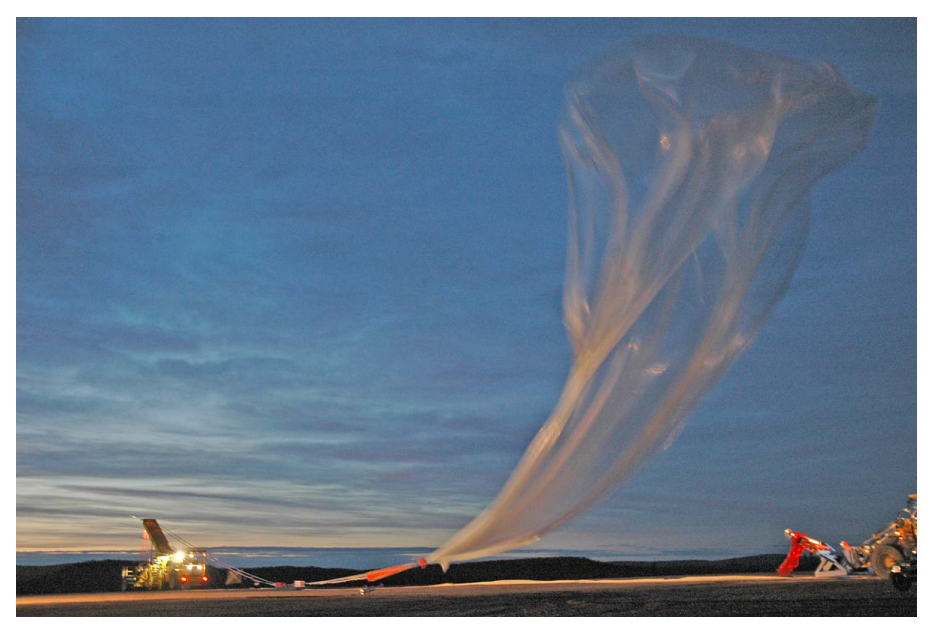

Fig. 3. Launch of the stratospheric ballon carrying the FELT payload 
Data was transmitted at rates of $622 \mathrm{Mbit} / \mathrm{s}$ and $1.25 \mathrm{Gbit} / \mathrm{s}$. Figure 4 shows an eye pattern and a Bit Error Rate (BER) that has been measured during the trial. Virtually error free data communication could be achieved with both data rates.

Furthermore, the turbulent behaviour of the atmosphere has been characterized with several measurement devices. A detailed description of the experiments carried out can be found in [4].

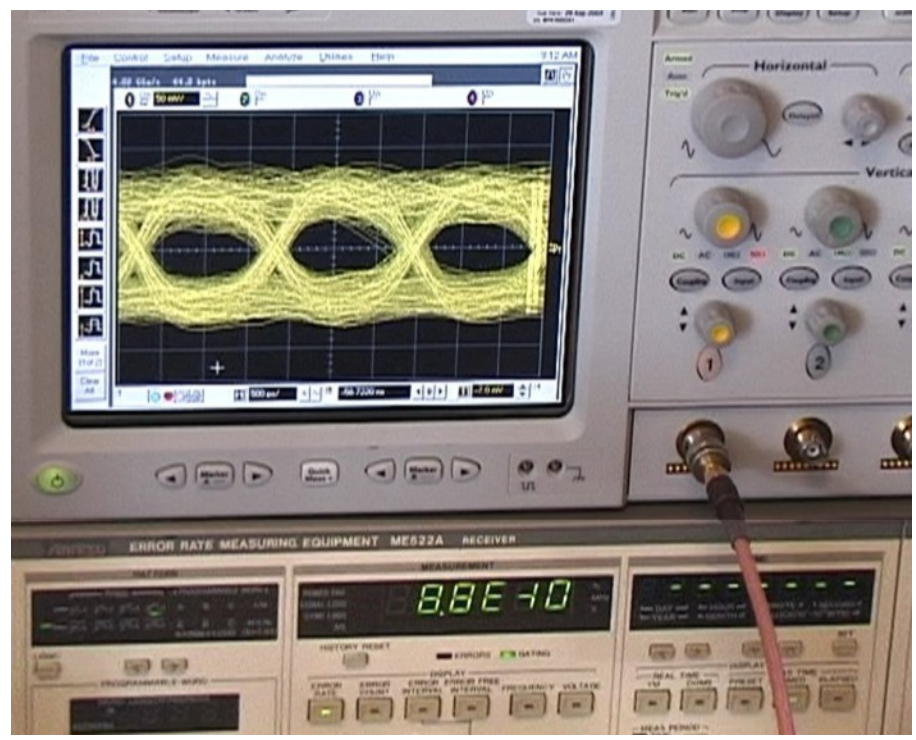

Fig. 4. Eye at the receiver and BER measured for a data rate of $622 \mathrm{Mbit} / \mathrm{s}$ during the CAPANINA trial

\section{Aircraft-to-Ground Links}

Optical Downlinks from aircraft have recently been demonstrated in DLR's ARGOS project. The ARGOS project (airborne wide area high altitude monitoring system) is meant to develop a platform to supply decision makers during massor even catastrophic events with real-time reconnaissance data of the current situation. The available sensors include a visible camera system and a synthetic aperture radar[5]. An optical downlink of the gathered data to a transportable optical ground station, placed at the operations center, is foreseen to obtain real-time data access.

In the frame of the project, an Airborne Optical Terminal for one of DLR's research aircraft has been developed. Its operability was shown over a link distance of up to $90 \mathrm{~km}$. The data rate for the system was driven by project requirements, and has been chosen to the rather low value of $125 \mathrm{Mbit} / \mathrm{s}$. Although this is a 
moderate data rate for FSO links, it is sufficient for the project requirements, and still greatly outperforms typical microwave links $(\approx 30 \ldots 40 \mathrm{Mbit} / \mathrm{s})$.

Figure 5 shows a picture of the terminal installed onboard the aircraft. Outside the aircraft, only the coude-type Coarse Pointing Assembly (CPA) is visible, while the rest of the terminal hardware is mounted inside the passenger cabin. Figure 6 shows the terminal in a laboratory and the Do228 aircraft during validation trials. Visible is the red marker laser, that was used for adjustment purposes. More information about the terminal design and its hardware can be found in $[6]$.
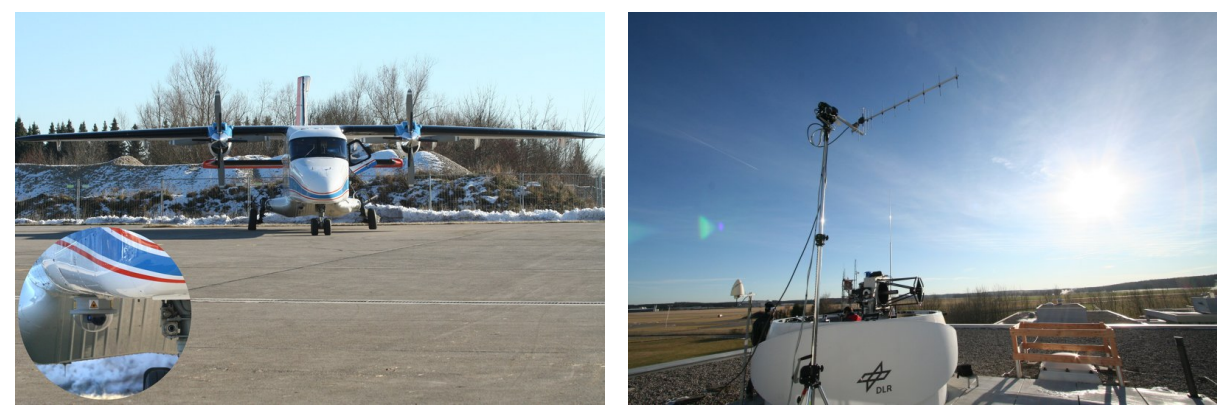

Fig. 5. Left: Picture of the Do228 Aircraft with installed Optical Terminal. Right: Picture of Optical Ground Station (OGS) with $40 \mathrm{~cm}$ Cassegrain Telescope for data reception and an RF link antenna for reception of GPS data from the aircraft
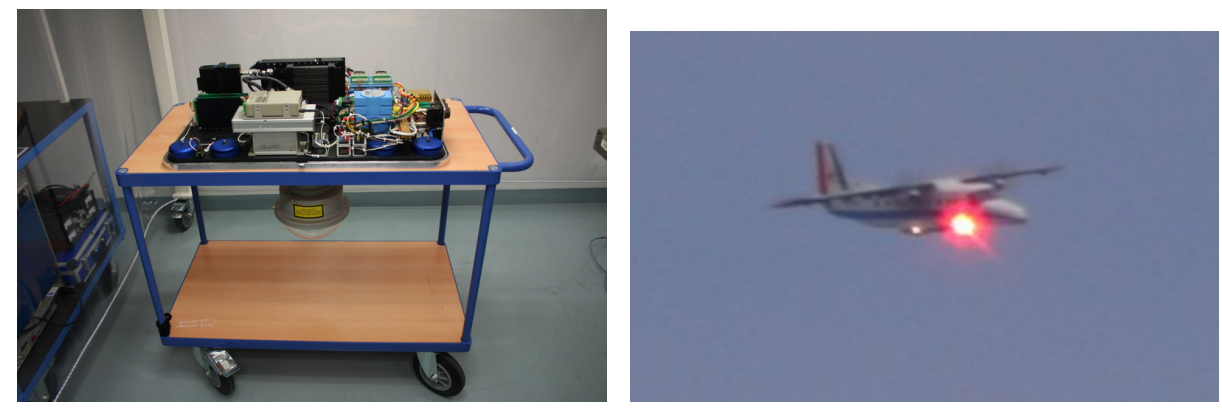

Fig. 6. Left: Optical Terminal in Lab. Right: View of Do228 aircraft during the trials. Visible is the red marker laser used for adjustment purposes.

Measurement results that were obtained for the beacon-uplink at the Airborne Terminal during trials in December 2008 are shown in Figure 7. The mean received power of approx. $15 \mathrm{nW}$ is consistent with prior link budget calculations, however, the signal shows a remarkable dynamic range. This is due to the fact that virtually no aperture averaging effect is existent for the comparatively small $30 \mathrm{~mm}$ aperture of the AT. 
The Pointing Error, that was calculated from the tracking camera's data, was most of the times below $1 \mathrm{mrad}$, with a mean of $266 \mu \mathrm{rad}$. This order of magnitude could be ascertained even during relatively fast and large changes of the aircraft's roll angle, visible in the bottom graph of Figure 7.
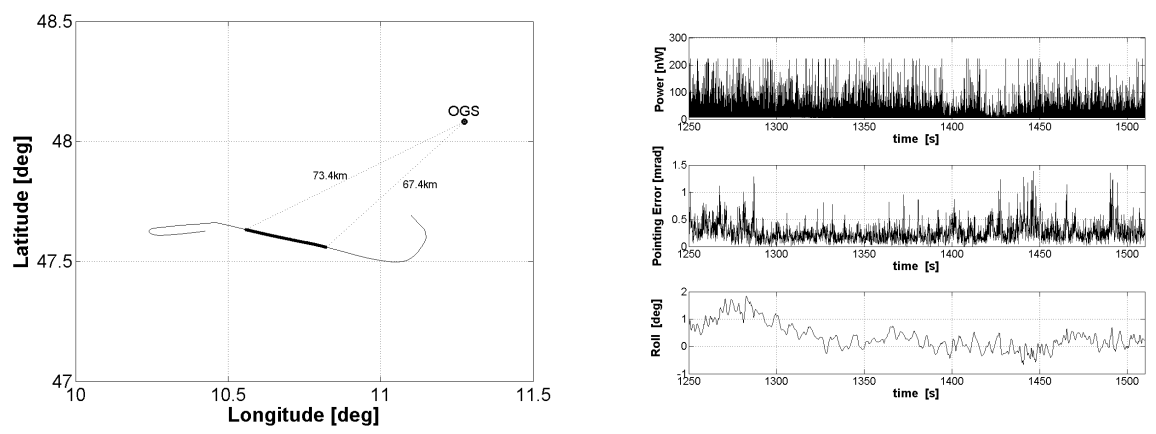

Fig. 7. Left: Flight Path example during validation flight. Right: Measurement data in the beacon-uplink for the thick painted line; Top: Received Power at Airborne Terminal, Middle: Pointing Error of Airborne Terminal, Bottom: Roll Angle of aircraft

Considering the beam divergence of $2 \mathrm{mrad}$, and taking into account the forward error correction that is applied to the sent data, an error free communication link can be established with this setup.

However, the development of the terminal is still ongoing. Possible further improvements include the implementation of a Fine Pointing Assembly (FPA), consisting of a fast steering mirror and a fast tracking sensor. With this new subsystem, a very fast tracking of the impinging beacon light would be possible, resulting in a stable tracking during agile flight maneuvers. Furthermore, the system can compensate for vibrations of the aircraft that are transferred to the terminal hardware.

The new developments will be verified by further flight testing campaigns. The ultimate goal of the project is to demonstrate an optical link over a distance higher than $100 \mathrm{~km}$ with a datarate of over $1 \mathrm{Gbit} / \mathrm{s}$.

\section{Satellite-to-Ground Links}

Also for the downlink of earth observation data gathered by LEO satellites, optical links to ground stations or High Altitude Platforms (HAPs) can be a solution for the increasing amount of downlink volume.

Typical RF Systems for high data rates in the X- and/or Ka-Band require tens to hundreds of Watts of electrical power and antenna diameters of several to tens of centimeters onboard the satellite. Furthermore, antenna dishes with several meters diameter are necessary on ground station side. It is believed 
that optical downlink terminals for LEO satellites can be built with lower power consumptions $(<50 \mathrm{~W})$ and smaller telescope diameters (few centimers). Furthermore, ground station telescope diameters in the range from $10 \mathrm{~cm}$ to $40 \mathrm{~cm}$ are sufficient and available at reasonable prices.

The largest drawback of optical downlink technology, link blocking by clouds, can be mitigated with ground station diversity concepts. It has been shown that availabilities of almost $100 \%$ can be achieved with four ground stations placed at beneficial locations throughout europe[7]. Further improvements of availability and downlink volume can be achieved with using HAPs as data relay platform $[8,1]$.

Successful downlink experiments from JAXA's OICETS satellite have been carried out to Tokio during the KODEN trials $[9,10]$. Further experiments have been carried out by DLR during the KIODO trials to Oberpfaffenhofen close to Munich[11]. OICETS uses a wavelength in the $800 \mathrm{~nm}$ range for communication, what is a very common wavelength for quantum communication systems. The data-rate was around $50 \mathrm{Mbit} / \mathrm{s}$.

In 2006, a total of eight trials have been performed to Oberpfaffenhofen, of which five were successful, while the others failed due to cloudy weather. Figure 8 shows the Optical Ground Station Oberpfaffenhofen (OGS-OP) with its $40 \mathrm{~cm}$ cassegrain telescope during the trials.

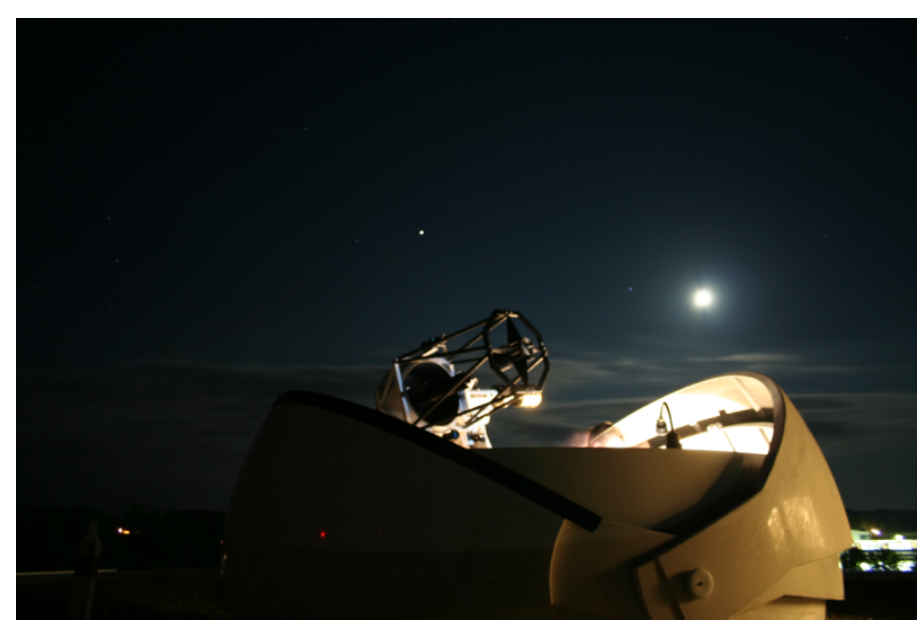

Fig. 8. Optical Ground Station Oberpfaffenhofen during KIODO Trials in 2006

As an example, Figure 9 shows the received power during KIODO Trial 3. It is visible that the power level increases while the fading gets lower during the trial. This is for the fact that the link distance through the atmosphere gets lower for higher elevation angles, resulting in a less turbulent communication channel and, of course, a higher power due to the shorter link distance. The visible signal 
breaks are characterstic of the laser terminal onboard OICETS[10], and don't appear due to mispointing of the telescope.

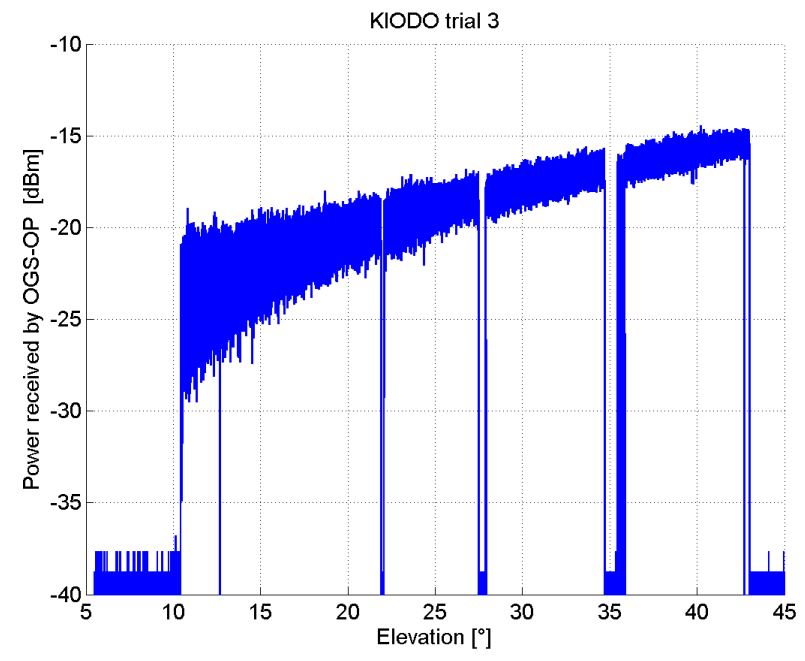

Fig. 9. Received Power during KIODO Trial 03[11]

Figure 10 shows the Bit Error Rate results that have been obtained during a selection of the KIODO trials. It is visible that the Bit Error Rates get lower for higher elevation angles, as the received power increases and the scintiallation due to atmospheric turbulence gets lower.

Further trials with OICETS were carried out during a seconds campaign in the summer of 2009. In addition to the previous trials, a shack-hartmann wavefront sensor was installed as additional measurement instrument. The gathered data will be used to evaluate the turbulent behaviour of the atmosphere, especially for low elevation angles (and thus high link distances). Furthermore, it will allow the simulation of adaptive optics systems and their theoretical performance in this very demanding scenario.

Besides this new measurement device, a data acquisition device with a sample-rate of five times the data-rate $(250 \mathrm{MBit} / \mathrm{s})$ was used to sample the signal at the output of the receiver frontend. By implementing the subsequent signal processing chain in a simulation program, it becomes possible to evaluate the Bit Error Rate results offline. With this information, the parameters of the receiver can be optimized for the purpose of designing an optimal receiver frontend for the turbulent atmospheric channel.

The data that was acquired during the 2009 trials is still under evaluation, however, it can already be stated that during all cloud-free links a signal from the satellite could be acquired and a data-communication link was be set up. However, again a number of links failed due to cloudy weather. Figure 11 show OGS-OP during the KIODO 2009 trials. 


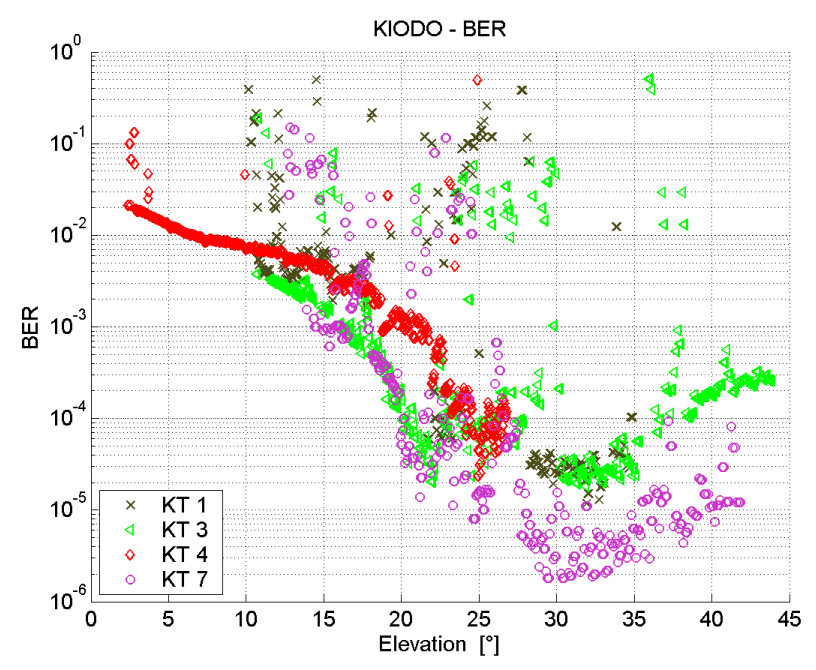

Fig. 10. Bit Error Rate Results for different elevation angles[11]

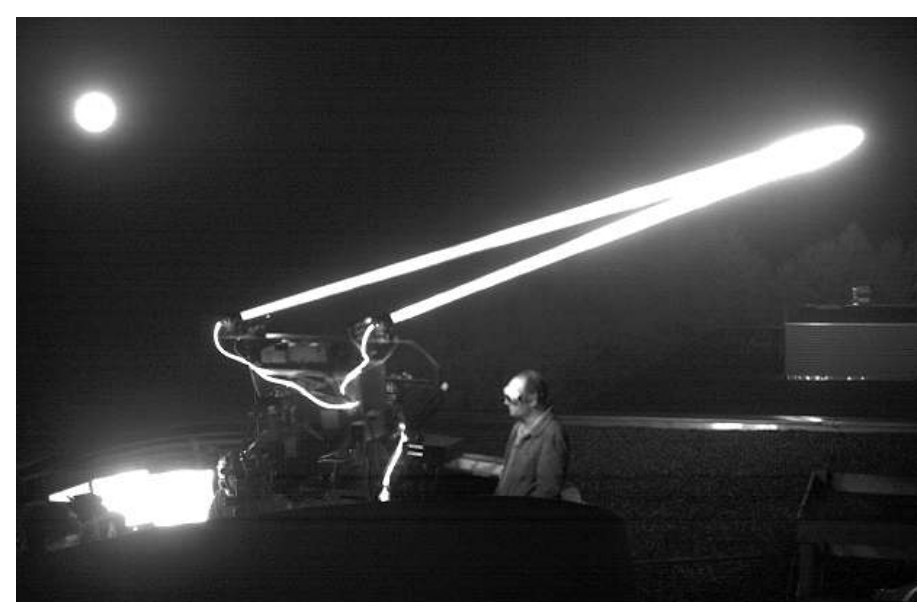

Fig. 11. Optical Ground Station Oberpfaffenhofen (OGS-OP) during the KIODO 2009 trials. Visible on the infrared picture is the beacon laser's backscatter due to haze.

The possibility of optical LEO downlinks for the transmission of e.g. earth sensing data using state-of-the art $1550 \mathrm{~nm}$ components is currently investigated at DLR. Figure 12 shows an example link budget for a downlink from a LEO satellite to the Optical Ground Station in Oberpfaffenhofen (OGS-OP) for two different aperture diameters with diffraction limited radiation from the satellite. Atmospheric attenuation based on [12] has been considered for clear sky conditions and moderate vulcanic activity.

It is visible that already with the small aperture of $10 \mathrm{~mm}$ onboard the satellite decent power levels can be achieved at the OGS, enabling data links with 


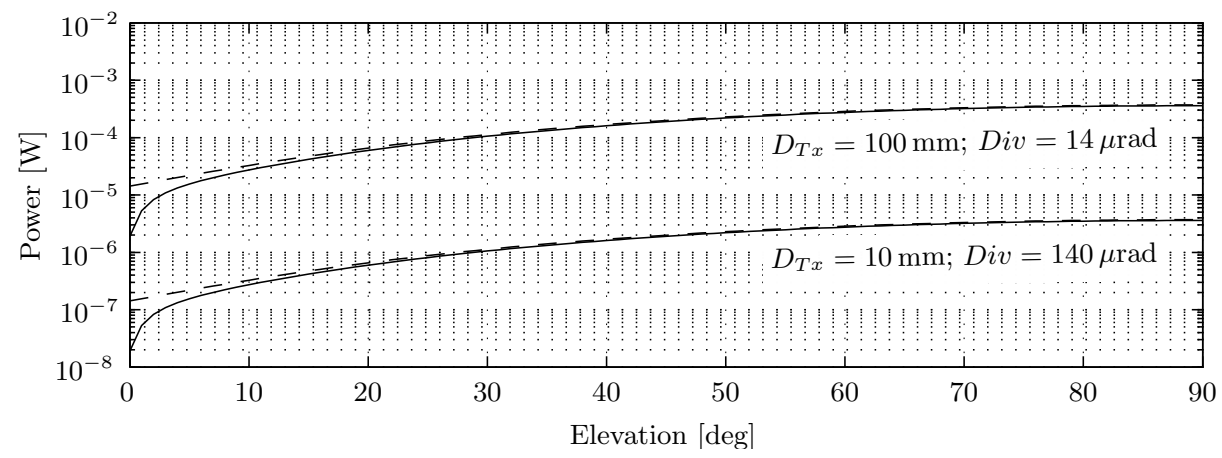

Fig. 12. Example Link-Budget for a diffraction limited optical LEO Downlink to OGSOP with atmospheric attenuation (solid line) and without (dashed line) for different Tx-Apertures; Link Parameters: 3 dB Rx-System Loss, 40 cm Rx-Aperture, Wavelength 1550 nm, Orbit Height 510 km, Clear Sky, Moderate Vulcanic Activity.

data rates from $100 \mathrm{Mbit} / \mathrm{s}$ up to more than $1 \mathrm{Gbit} / \mathrm{s}$, depending on the sensitivity of the receiver. However, it is remarkable that the influence of the atmospheric attenuation increases drastically for elevation angles close to 0 , as for these cases the path through the atmosphere is very long and close to a horizontal link with worst-case attenuation conditions.

\section{Further Developments}

The research field of free-space optical communications offers still many open questions that are worth investigating them. One topic that is currently researched at DLR is e.g. an adaptive optics system fitted to the particular needs of FSO systems operating in the turbulent atmosphere. A system consisting of a wavefront sensor and a deformable mirror can be used to mitigate the influences of the atmosphere on the communication beam. A first lab-setup of this challenging development is intended to be operational in 2010 .

Another interesting topic for further research is the application of FSO links for Deep-Space communications. With the inherent advantages of Optical Communications, as e.g. the reduced beam divergence, lower antenna size and lower power consumption, data rates from remote places in the solar system could be increased, by relaxing the power budget for Space Probe Missions at the same time.

Currently, development and qualification actions are carried out for the purpose of developing a small and lightweight optical terminal for the deployment on small- and micro- LEO satellites. It is foreseen to use the extremely precise attitude control systems used on these satellites to point the communication laser beam towards the ground station by rotating the satellite. Thus no bulky Coarse Pointing Assembly is necessary for the beam pointing, resulting in a very 
power-efficient and light-weight optical terminal with high data rates. In-Orbit validations of this promising development will be accomplished in the future.

\section{Applicability of Quantum Cryptography to Standard FSO Links}

In principle, the communication subsystems of the mentioned optical terminals can be modified for using them in quantum communication applications. A possible design of a combined optical transceiver for traditional FSO- and quantum communications using state-of-the-art technology has e.g. been proposed in [13]. Corresponding developments are already carried out - for instance, the SpaceQUEST initiative is aiming at the installation of a quantum communication terminal onboard the ISS[14, 15].

Common FSO terminals for communication purposes operate at a wavelength of $1550 \mathrm{~nm}$. The atmospheric transmission at this wavelength is more benefical than for shorter wavelengths in the $800 \mathrm{~nm}$ region, as they are typical for quantum communication systems. This effect is illustrated in Figure 13 for the example of an aircraft-to-ground link.

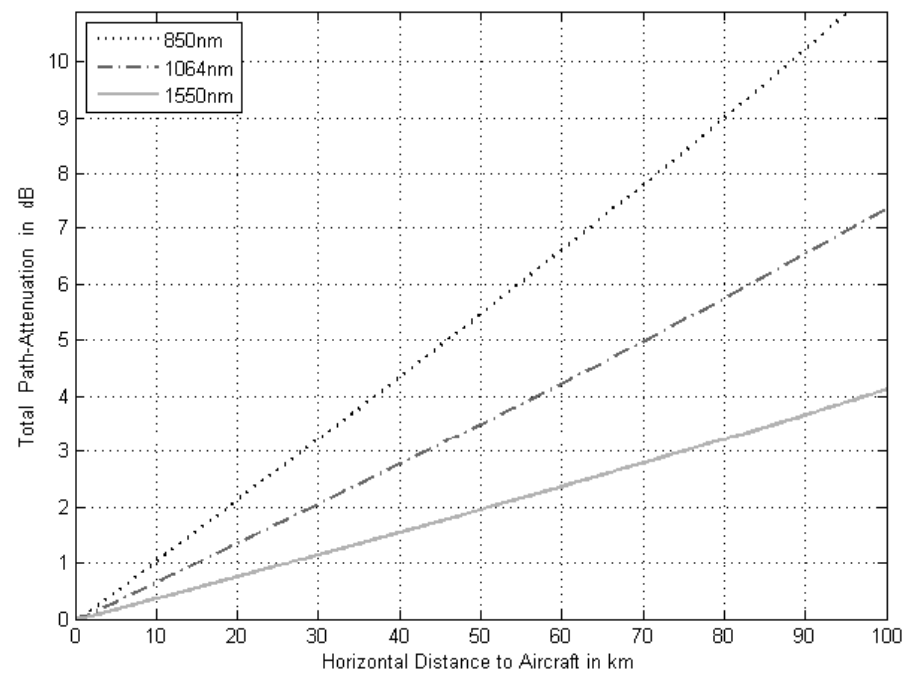

Fig. 13. Different atmospheric attenuations for an aircraft-to-ground link. It is visible that systems with longer wavelengths can take advantage of lower atmospheric attenuations[16]

For the relatively short link distances of optical aircraft-to-ground links, there is no need to design the communication system close to the diffraction limit. Instead, larger beam divergences can still fulfill the project requirements in terms of free-space loss and thus data-rate, with the advantage that the requirements 
for the tracking system can be relaxed and a less accurate tracking system is sufficient. Furthermore, many mature and low prized off-the-shelf components are available in the $1550 \mathrm{~nm}$ range.

However, for system designs close to or at the diffraction limit, as e.g. for satellite downlinks, the lower possible beam divergences of shorter wavelengths can compensate for the higher atmospheric attenuation. An example link budget for an optical downlink visualizing this effect is depicted in Figure 14. As for Figure 12, atmospheric attenuation based on [12] has been considered.

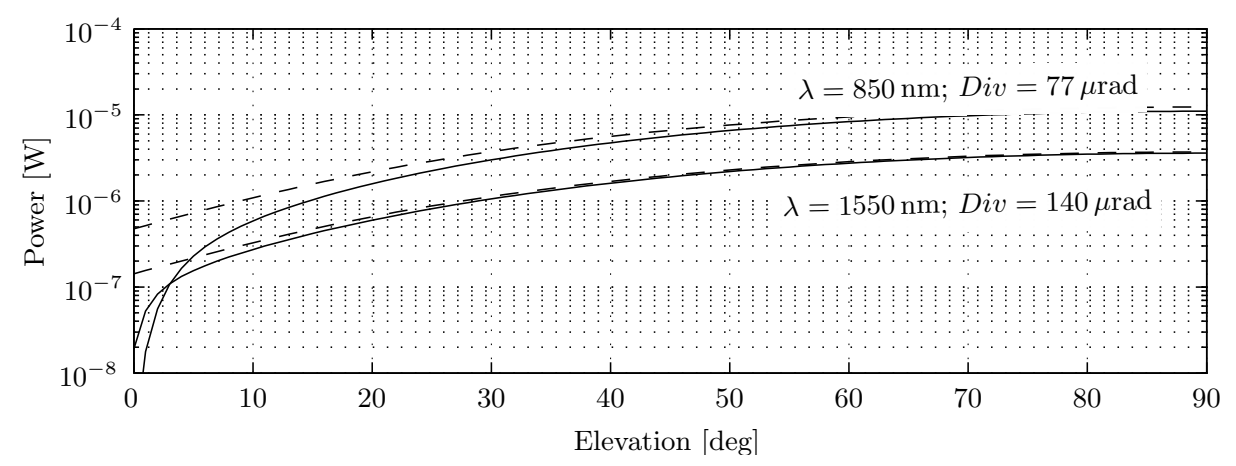

Fig. 14. Example Link-Budget for a diffraction limited optical LEO Downlink to OGSOP with atmospheric attenuation (solid line) and without (dashed line) for different wavelengths; Link Parameters: $3 \mathrm{~dB}$ Rx-System Loss, $10 \mathrm{~mm}$ Tx-Aperture, $40 \mathrm{~cm}$ RxAperture, Orbit Height 510 km, Clear Sky, Moderate Vulcanic Activity.

It is visible, that the better atmospheric attenuation for a $1550 \mathrm{~nm}$ system has only an effect for very low elevation angles. For any elevation angle over 5 degrees, the received power is higher for an $850 \mathrm{~nm}$ system.

Due to the mentioned component availability and -maturity, $1550 \mathrm{~nm}$ is still a good choice for communication systems. However, considering a combination of a classical FSO- and of a quantum communication terminal, the tracking could be accomplished with the $1550 \mathrm{~nm}$ wavelength. As the beam divergence for this system would be larger, tracking requirements would be somewhat relaxed. A quantum communication system in the $800 \mathrm{~nm}$ region could be included in the same system, as the splitting between the wavelengths for communication and quantum key exchange could be accomplished with ease and by means of standard components.

In such a combined system, the same aperture could be used for both systems. Thus, only one tracking system is necessary and the extension of a standard FSO communication terminal to a combined FSO-/Quantum communication terminal can be accomplished with relatively little effort. This procedure would allow the tap-proof exchange of an encryption key at the beginning of a data communication session, e.g. for a downlink from LEO satellites. Especially for 
the direct downlink of sensible data from LEO- or GEO satellites, such a kind of communication terminal would be a promising development.

\section{Conclusions}

Optical Free-Space Communications offer a solution for the continuously increasing demand of higher data rates in many applications. The German Aerospace Center's Institute of Communication and Navigation's Optical Communication Group pursues research concerning terminal design and characterization of the atmospheric influences on FSO links for many of these applications.

The feasibility of FSO links has been shown for a diverse spectra of applications. This includes the execution of validation trials from stratospheric platforms, aircrafts and satellites.

Furthermore, it seems feasible to comprise both standard FSO- and quantum communication subsystems into a combined optical terminal. This procedure would allow the establishment of tap-proof communication links over very high link distances, combining the advantages of both worlds: The tap-proof exchange of encryption keys with quantum communications, and the very high data-rates and power efficiencies of FSO links.

The next step towards an introduction of this combined technology would be the development of a technology demonstrator. Similar to the mentioned SpaceQUEST initiative, the demonstator could be aimed at showing it's functionality with an actual LEO downlink from either the ISS, or another suitable LEO satellite.

\section{Acknowledgement}

The authors wish to thank all members of the Optical Communication Group for their efforts and dedication, without whose the activities mentioned above would not have been possible. Furthermore, the support of JAXA during the KIODO-trials is gratefully appreciated.

\section{References}

1. M. Knapek et al. Optical high-capacity satellite downlinks via high-altitude platform relays. In Proceedings of the SPIE, 2006.

2. D. Giggenbach and J. Horwath. Optical free-space communications downlinks from stratospheric platforms - overview on stropex, the optical communications experiment of capanina. In IST Summit Dresden, 2005.

3. J. Horwath et al. Broadband backhaul communication for stratospheric platforms: The stratospheric optical payload experiment (stropex). In Proceedings of the SPIE, 2006. 
4. M. Knapek et al. The DLR ground station in the optical payload experiment (stropex) - results of the atmospheric measurement instruments. In Proceedings of the SPIE, 2006.

5. F. Kurz et. al. Near real time airborne monitoring system for disaster and traffic applications. In ISPRS Hannover Workshop, 62009.

6. J. Horwath and C. Fuchs. Aircraft to ground unidirectional laser-comm. terminal for high resolution sensors. In Free-Space Laser Communications Technologies XXI, San Jose, USA, 01 2009. SPIE.

7. F. Moll and M. Knapek. Wavelength selection criteria and link availability due to cloud coverage statistics and attenuation affecting satellite, aerial, and downlink scenarios. In Proceedings of the SPIE, 2007.

8. D. Giggenbach et al. Optical satellite downlinks to optical ground stations and high-altitude platforms. In IST Mobile ${ }_{6}$ Wireless Communication Summit, 2007.

9. M. Toyoshima et al. Ground-to-oicets laser communication experiments. In Proceedings of the SPIE, 2006.

10. Y. Takayama et al. Tracking and pointing characteristics of oicets optical terminal in communication demonstrations with ground stations. In Proceedings of the SPIE, 2007.

11. N. Perlot et al. Results of the optical downlink experiment kiodo from oicets satellite to optical ground station oberpfaffenhofen (ogs-op). In Proceedings of the SPIE, 2007.

12. B. Mayer et al. Dlr-internal electronic data base of atmospheric absorption coefficients. In DLR internal, 2002.

13. M. Pfennigbauer et al. Satellite-based quantum communication terminal employing state-of-the-art technology. Journal of Optical Networking (JON), 4, 2005.

14. R. Ursin et al. Space-quest: Experiments with quantum entanglement in space. Europhysicsnews, 40(3), 2009.

15. Josep Maria Perdigues Armengol et al. Quantum communications at ESA: Towards a space experiment on the iss. In 58th International Astronautical Congress, Hyderabad, India, 2007.

16. D. Giggenbach. Mobile optical high-speed data links with small terminals. In Proceedings of the SPIE, 2009. 